\title{
Anti-IgE (Omalizumab) in the Management of ABPA among Cystic Fibrosis Patients: An Alternative to Steroid Treatment?
}

\author{
Marianne Skov* \\ CF Center, National University Hospital, Rigshospitalet, Denmark
}

*Corresponding author: Marianne Skov, CF Center, National University Hospital, Rigshospitalet, Blegdamsvej 9, 2100 Copenhagen $\varnothing$, Denmark

Airway colonization with Aspergillus species, typically $A$. fumigatus, is frequent among cystic fibrosis (CF) patients. A subgroup of the patients develops allergic bronchopulmonary aspergillosis (ABPA) - an immunological reaction directed against the fungal infection with serious clinical consequences for the patients. Between one and $15 \%$ of the CF population is reported to be affected by ABPA [1]; The optimal treatment for APBA has not been clearly identified. Generally, steroid treatment, to tapper the immunological overreaction, is combined with azoles, to minimize the level of molds feeding the process [2]. At the Copenhagen CF Centre series of monthly intravenous bolus treatments of steroids (HDIVMP) have been preferred over continuously oral steroid treatment [3-5]. Some patients at our clinic have declined this treatment due to significant side effects (mood swings, difficulties with concentration af- fecting performance at school/work etc.). Furthermore, we have been reluctant to use this treatment strategy in patients with concurrent active non-tuberculous mycobacterial infection (NMT) [6].

The search for alternative efficient and safe therapeutic options for this patient group led to the initiation of an exploratory study of Omalizumab for CF patients with ABPA (NCT00787917). Omalizumab is a humanized recombinant monoclonal antibody directed against IgE and prevents its binding to receptors on effector cells mediating inflammation and a hypersensitivity immune response.

Unfortunately, the first study was prematurely stopped due to difficulties with recruitment. However, several reports, although universally with few patients, have reported positive results of Omalizumab treat-

Table 1: Demography.

\begin{tabular}{|l|l|l|l|l|}
\hline Pt no & $\begin{array}{l}\text { Sexe } \\
\text { M/F }\end{array}$ & $\begin{array}{l}\text { Age } \\
\text { years }\end{array}$ & $\begin{array}{l}\text { Lung infection } \\
\text { other than Aspergillus } \\
\text { chronic PA }\end{array}$ & Inclusion criteria \\
\hline 1 & M & 17 & chronic S. maltophilia, NTM ${ }^{\#}$ & side-effects to HDIVPM \\
\hline 2 & F & 14 & intermittent PA & side-effects to HDIVPM \\
\hline 3 & M & 16 & NTM & NTM \\
\hline 4 & M & 17 & chronic Inquilinus limosus & side-effects to HDIVPM \\
\hline 5 & M & 18 & chronic PA & side-effects to HDIVPM \\
\hline 6 & M & 36 & chronic PA & side-effects to HDIVPM \\
\hline 7 & M & 19 & & \\
\hline & & mean 19.6 & & \\
\hline
\end{tabular}

"PA: Pseudomonas aeruginosa; \#NTM: Non-tuberculous mycobacteria; \$HDIVPM: High-dose i.v. pulse methylprdnisolon.

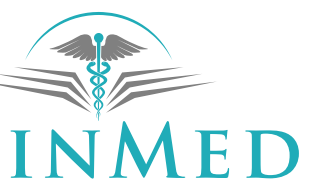

INTERNATIONAL LIBRARY

Citation: Skov M (2019) Anti-IgE (Omalizumab) in the Management of ABPA among Cystic Fibrosis Patients: An Alternative to Steroid Treatment?. Int J Aller Medications 5:039. doi.org/10.23937/25723308.1510039

Accepted: May 23, 2019: Published: May 25, 2019

Copyright: (c) 2019 Skov M. This is an open-access article distributed under the terms of the Creative Commons Attribution License, which permits unrestricted use, distribution, and reproduction in any medium, provided the original author and source are credited. 
ment for CF-related ABPA [7-9]. In the reported cases, in addition to clinical as well as improvement in FEV1, steroid sparing has been used as an efficacy parameter.

The present report describes a pilot study of Omalizumab treatment of APBA-inflicted patients in the Copenhagen CF Centre. Seven CF patients with ABPA and contraindication for HDIVPM (Table 1) were treated with Omalizumab. In addition to lung function (FEV1 and FVC), total IgE, Aspergillus-specific IgE and IgG, eosinophil count, we measured Aspergillus specific histamine release (HR) [10] in order to investigate its applicability to monitor treatment.

The patients had previously been treated with HDIVMP for at least one year. All the patients are homozygous for delta F508, and three of seven patients have chronic $P$. aeruginosa (PA) pulmonary infection (Table 1). Omalizumab was given every second week three times, followed by every fourth week for another three times (between 300-600 mg - weight and IgE level dependent - according to manufacturer's instructions - , max $150 \mathrm{mg}$ at each injection site). After 16 weeks the treatment was evaluated. All of the patients received concomitant treatment with oral azoles.

As illustrated in Figure 1, lung function, FEV1 and FVC, remained stable during treatment. Total IgE increased during treatment, whereas Aspergillus-IgG-and-IgE varied among patients. Total IgE as well as Aspergillus-specific IgE and IgG levels had decreased at follow-up (6-8 months after treatment stopped) as compared to pre-treatment levels. Eosinophil counts increased during treatment in two patients, but in the remaining five patients the counts were unchanged and within normal range (data not shown). Aspergillus-HR levels varied. There was a decrease during treatment in only two of seven patients. Follow-up levels showed a great variation. None of the patients were hospitalized within the study period.

As expected total IgE increased during treatment [11]. None of the previous Omalizumab studies monitored specific Aspergillus IgE and IgE antibodies. We found varying results with increase in specific antibody levels in some patients and decrease in others. However, similar to total IgE, the levels had decreased at follow-up as compared to pre-treatment levels, indicating a positive response to Omalizumab. In our study set up steroid-sparing could not be used as a monitoring parameter. The intervals between the first treatment doses (two weeks) prescribed in our study was shorter as compared to other studies (one month). Whether this influences the effect or onset of effect is unknown. In previous studies of Omalizumab lung function was not stable until after six months of treatment $[9,12]$. In our patients effect on lung function is difficult to evaluate because they had a stable lung function due to HDIVMP. However, lung function remained stable during Omalizumab treatment. HDIVMP was re-started in four pa-
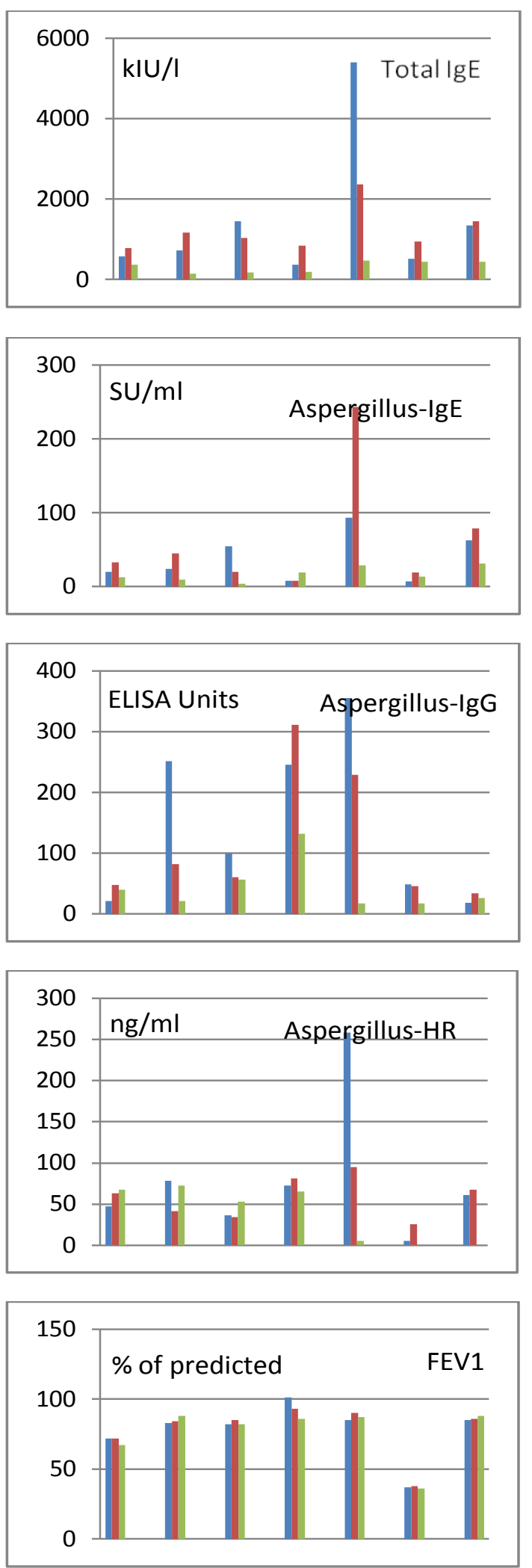

$\begin{array}{llllllll}\text { Patient } & 1 & 2 & 3 & 4 & 5 & 6 & 7\end{array}$

Serie 1: Treatment start.

Serie 2: Treatment stop.

Serie 3: Follow-up, 6-8 months after treatment stop.

Figure 1: Lung function and serological parameters at treatment start, stop and follow-up in seven CF patients with ABPA treated with Omalizumab. 
tients, in two patients due to clinical deterioration and in the other two patients due to persistent elevation of serological markers. It may, however, be speculated whether the observation period before re-start was sufficient. Another patient has refused to stop Omalizum$a b$, and the remaining two patients are still at remission stage after one year. A prolonged study period is needed to evaluate time to exacerbation of ABPA.

Omalizumab has previously been reported to be efficient in asthmatic patients [7] as well as CF patients [8,9,13-15] with ABPA as steroid saving agent [7$9,16,17]$ reducing ABPA exacerbations [8], stabilizing lung function $[9,15]$ and/or reducing hospitalization rate [8].

Serum IgE is typically used a marker of exacerbation of ABPA. Omalizumab blocks the binding of specific IgE on mast cells and basophils, and thereby prevents degranulation. An increased half-life of circulating IgE-Omalizumab complexes results in elevated IgE during treatment. However, free IgE level declines, and has been used to monitor treatment [7]. Alternatively to free IgE, which is difficult to measure, we measured Aspergillus histamine release, but failed, in our shortterm treatment regimen, to prove it suitable as a monitor of Omalizumab therapy.

HDIVPM has, despite efficacy and priority to oral glucocorticosteroids, to be avoided in some patients. We found that Omalizumab in selected cases can be used in combination with azoles but without corticosteroids as an alternative treatment in ABPA. With an emerging prevalence of NTM in CF patients [12], reluctance to treat with steroids, and more frequent isolation of Aspergillus among these patients [18], alternative ABPA treatments are needed. Placebo controlled, long-term trials are still needed as well as improvement of monitoring tools in the treatment of ABPA in CF.

\section{References}

1. Stevens DA, RB Moss, VP Kurup, AP Knutsen, $P$ Greenberger, et al. (2003) Allergic bronchopulmonary aspergillosis in cystic fibrosis--state of the art: Cystic Fibrosis Foundation Consensus Conference. Clin Infect Dis 37: S225-S264.

2. Skov M, N Hoiby, C Koch (2002) Itraconazole treatment of allergic bronchopulmonary aspergillosis in patients with cystic fibrosis. Allergy 57: 723-728.

3. Cohen-Cymberknoh M, H Blau, D Shoseyov, M MeiZahav, O Efrati, et al. (2009) Intravenous monthly pulse methylprednisolone treatment for ABPA in patients with cystic fibrosis. J Cyst Fibros 8: 253-257.

4. Thomson JM, A Wesley, CA Byrnes, GM Nixon (2006) Pulse intravenous methylprednisolone for resistant allergic bronchopulmonary aspergillosis in cystic fibrosis. Pediatr Pulmonol 41: 164-170.

5. Skov M, T Pressler (2012) Highdose iv methylprednisolone (HDIVPM): Successful treatment of allergic bronchopulmonary aspergillosis (ABPA). Pediatr Pulmonol.

6. Hoefsloot W, IJ van, EJ Peters, C Magis-Escurra, PN Dekhuijzen, et al. (2013) Mycobacterium genavense in the Netherlands: An opportunistic pathogen in HIV and nonHIV immunocompromised patients. An observational study in 14 cases. Clin Microbiol Infect 19: 432-437.

7. Collins J, G Devos, G Hudes, D Rosenstreich (2012) Allergic bronchopulmonary aspergillosis treated successfully for one year with omalizumab. J Asthma Allergy 5: 65-70.

8. Elmallah MK, L Hendeles, RG Hamilton, C Capen, PM Schuler (2012) Management of patients with cystic fibrosis and allergic bronchopulmonary aspergillosis using anti-immunoglobulin e therapy (omalizumab). J Pediatr Pharmacol Ther 17: 88-92.

9. Wong R, M Wong, PD Robinson, DA Fitzgerald (2012) Omalizumab in the management of steroid dependent Allergic Bronchopulmonary Aspergillosis (ABPA) complicating Cystic Fibrosis. Paediatr Respir Rev 14: 2224.

10. Skov PS, H Mosbech, S Norn, B Weeke (1985) Sensitive glass microfibre-based histamine analysis for allergy testing in washed blood cells. Results compared with conventional leukocyte histamine release assay. Allergy 40: 213-218.

11. Nowak D (2006) Management of asthma with antiimmunoglobulin E: A review of clinical trials of omalizumab. Respir Med 100: 1907-1917.

12. Catherinot $E, A L$ Roux, MA Vibet, G Bellis, L Lemonnier, et al. (2013) Inhaled therapies, azithromycin and Mycobacterium abscessus in cystic fibrosis patients. Eur Respir J 41: 1101-1106.

13. Zirbes JM, CE Milla (2008) Steroid-sparing effect of omalizumab for allergic bronchopulmonary aspergillosis and cystic fibrosis. Pediatr Pulmonol 43: 607-610.

14. Lebecque P, A Leonard, C Pilette (2009) Omalizumab for treatment of ABPA exacerbations in CF patients. Pediatr Pulmonol 44: 516.

15. Tanou K, E Zintzaras, AG Kaditis (2014) Omalizumab therapy for allergic bronchopulmonary aspergillosis in children with cystic fibrosis: A synthesis of published evidence. Pediatr Pulmonol 49: 503-507.

16. Beam KT, CA Coop (2015) Steroid sparing effect of omalizumab in seropositive allergic bronchopulmonary aspergillosis. Allergy Rhinol (Providence.) 6: 143-145.

17. Zicari AM, C Celani, CG De, BR Valerio De, M Duse (2014) Anti lgE antibody as treatment of allergic bronchopulmonary aspergillosis in a patient with cystic fibrosis. Eur Rev Med Pharmacol Sci 18: 1839-1841.

18. Catherinot E, AL Roux, MA Vibet, G Bellis, S Ravilly, et al. (2013) Mycobacterium avium and Mycobacterium abscessus complex target distinct cystic fibrosis patient subpopulations. J Cyst Fibros 12: 74-80. 